\title{
Report on the Digital Ludeme Project
}

\author{
Dennis J.N.J. Soemers, Walter Crist and Cameron Browne* \\ Department of Data Science and Knowledge Engineering, Maastricht University, Maastricht, \\ The Netherlands
}

\begin{abstract}
This report summarises the discussions and conclusions of a recent Dagstuhl research meeting, in which the foundations of the new research field of Digital Archæoludology were established. Starting with the ERC-funded Digital Ludeme Project, research in this field aims to use techniques from the field of Artificial Intelligence (AI), and other computational and data-driven techniques, to study traditional games. The primary goals include gaining improved insight into the ways games may have been played throughout history, and how they influenced and transferred between different civilisations. Additional expected contributions of the Digital Ludeme Project include advances in General Game Playing and related areas of research in AI.
\end{abstract}

\section{INTRODUCTION}

Most human civilisations throughout history have played various types of games, such as board games, card games, dice games, etc. For many such games, evidence of how they were played and transferred between cultures is sparse. The ERC-funded Digital Ludeme Project ${ }^{1}$ (Browne, 2018, 2019) has initiated the new research field of Digital Archæoludology (DAL), which aims to use modern Artificial Intelligence (AI) techniques, and other computational and data-driven techniques, to generate new hypotheses and plausible reconstructions based on such sparse data. Leading experts in relevant fields such as history, archaeology, AI, mathematics, etc. were gathered in a Dagstuhl research meeting to establish the foundations of DAL (Browne et al., 2019). This report summarises some of the primary points discussed at this meeting, by providing an overview of what types of evidence of gameplay through history exist, and how AI techniques may be used to provide new insights.

\section{HISTORICAL CONTEXT OF GAMES}

Historical information about games can be gathered through the use of multiple different kinds of sources which provide varying kinds of data. One of the important aspects of historical games is that the rules were rarely written down; they typically were learned from another person. While this provides difficulties for historical game reconstruction, information can be gleaned from other sources which can provide crucial details about how, where, and by whom games were played.

In some cases, historical documents can provide details about game rules. One famous example is the Libro de los Juegos by Alfonso X, a compilation of the rules of games played around the year 1283 $\mathrm{CE}$, including the earliest known explanation of chess rules in Europe. In another case, an epigram

\footnotetext{
${ }^{*}$ Corresponding author. E-mail: cameron.browne@ maastrichtuniversity.nl.

${ }^{1}$ http://www.ludeme.eu/
} 


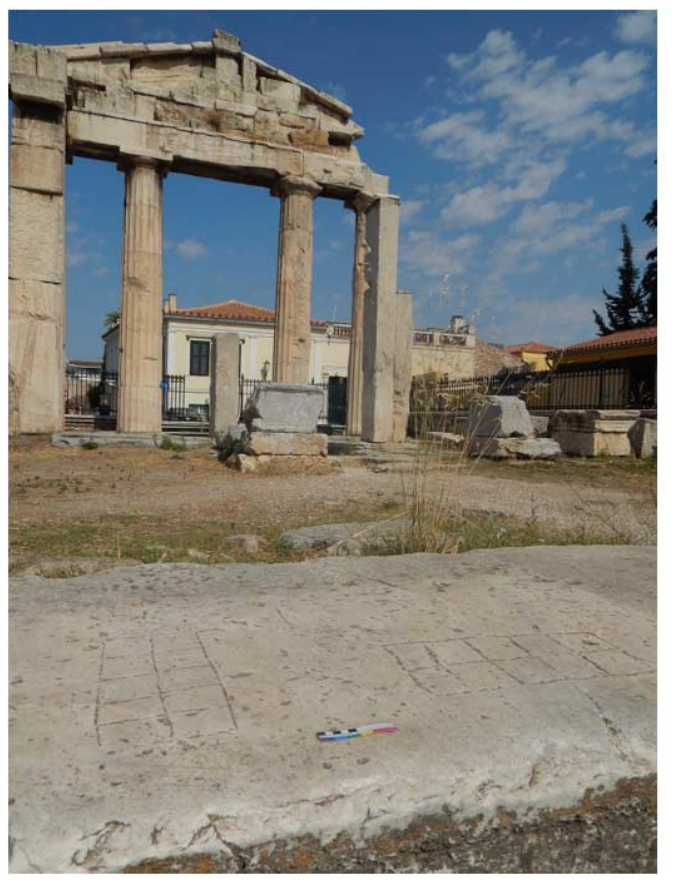

Fig. 1. Two pente grammai ("five lines") games from the Roman Agora in Athens ( $1^{s t}-7^{\text {th }}$ century CE). Aside from showing the layout of the board, their presence here shows that they were played in outdoor public spaces where many people gathered.

about the Byzantine emperor Zeno (r. 474-475 CE) (Greek Anthology 9.482), describes the moves the emperor made during a game called tabula, indicating that it was a form of backgammon.

Artistic depictions of people playing can also provide evidence about games. When visual representations of game boards are present, it is possible to document the presence of a certain game in particular locations or time periods. Sometimes they can provide information about rules as well, as in the case of scenes of people playing Egyptian senet games, which often include captions containing exclamations made by the players about the moves they are making (Piccione, 1990).

Archaeological evidence takes many forms that provide considerable data on the presence of games in different cultures, as well as who played them and, sometimes, about how they were played. Ancient gaming materials such as boards, playing pieces, and dice or other randomisation devices are important pieces of evidence for documenting ancient games, but these artefacts themselves merely provide the starting point for the understanding of ancient games. Archaeological context, information about the precise location that an object was found as well as other artefacts found associated with it, is crucial to archaeological inquiry as it provides data that allows for precise dating and interpretations of past human activity (see Fig. 1). The discovery of game boards in well-documented archaeological contexts can not only attest to the presence of games in a location or culture, but can also provide information on the places where people played (Crist, 2019), and with whom they were playing. All of these factors are crucial to understanding the ways in which games influence and travel between cultures. In some cases, the artefacts themselves can provide evidence for how games were played, such as when randomisation devices are found with sets of particular games. This is demonstrated in the tomb of Tutankhamun, which had two different types of randomisation devices contained in the drawers of boxes that had senet and the game of twenty squares on the same game box (Tait, 1982). On some ancient boards there are markings on the playing surface which likely indicate an outcome 
associated with moving a piece to that particular location on the board, providing further indications for how the game was played.

Finally, ethnographic accounts of game playing over the last 150 years can provide information on games played in societies worldwide, some of which are no longer played, while others are still played but may have changed. Though infrequently documented by anthropologists, when games are discussed they can provide information on how, where, and during what times games were typically played, as well as who was allowed to play them (e.g., were they restricted by sex or age) (Herskovits, 1932; Malaby, 2003). Games are most commonly mentioned in passing in ethnographic work, and this evidence can help to situate certain games in particular cultures. Nevertheless, comprehensive work done by Townshend (1982) and de $\operatorname{Voogt}(2003,1997)$ on mancala games are examples where the ethnographic documentation of the play of games provides valuable data useful for this research.

When used in concert, these lines of evidence can provide a strong case for the reconstruction of games past and present. Nevertheless, the nature of the data which are available is such that they are often incomplete, leaving room for revision and reinterpretation. Using computational techniques to fill in some of the gaps in our knowledge is valuable for furthering our understanding of ancient play and can provide insights into the social life of people that may be otherwise unattainable.

\section{LUDII GENERAL GAME SYSTEM}

To facilitate the efficient modelling of the wide variety of games studied in DAL, the Ludii (Piette et al., 2019b; Stephenson et al., 2019) general game system and its ludeme-based Game Description Language (GDL) are in development. It models games as trees of ludemes, which may intuitively be understood as conceptual units of game-related information (Parlett, 2016; Browne et al., 2019, Appendix A). An example Ludii game description, for the game of Tic-Tac-Toe, is depicted in Fig. 2.

Ludii has been demonstrated to be competitive with other commonly-used general game systems, such as the ones based on Stanford's GDL (Love et al., 2008) or the Regular Boardgames language (Kowalski et al., 2019), in terms of computational efficiency (Piette et al., 2019a). Due to the succinctness of Ludii's game descriptions, and the close relationship between ludemes and human-understandable, high-level game concepts, it may also be argued that Ludii's game descriptions are easier to read and write. Browne (2009) previously also demonstrated that ludeme-based approaches for modelling games allow effective evolution of new (variants of) games.

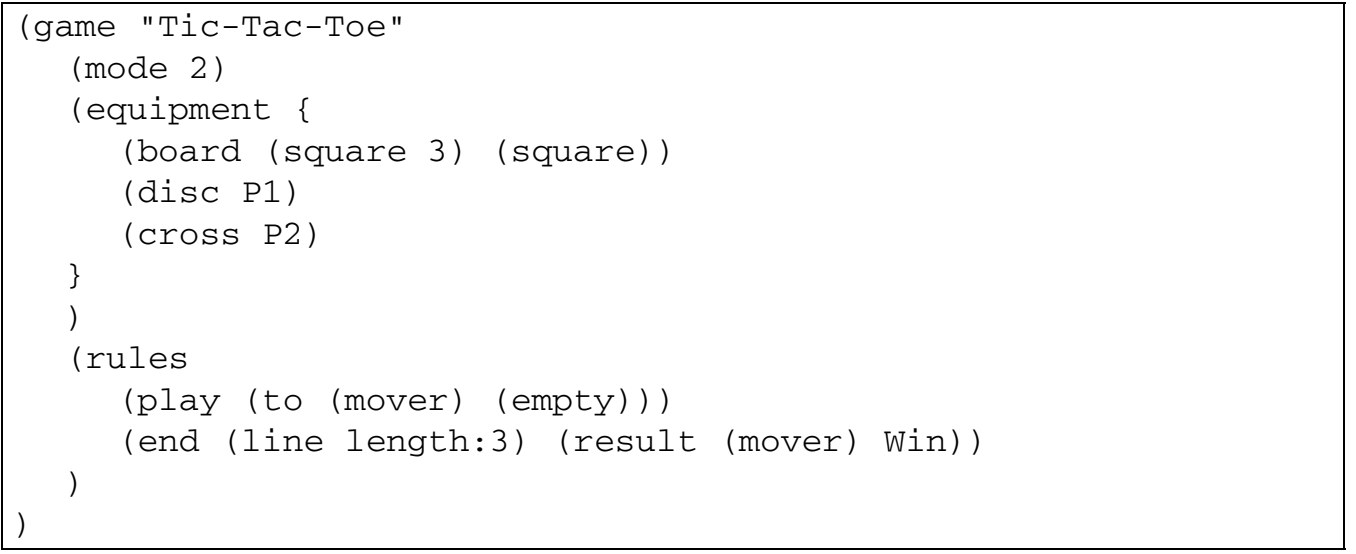

Fig. 2. The game of Tic-Tac-Toe modelled in Ludii. 


\section{AUTOMATED GAME ANALYSIS AND RECONSTRUCTION}

One example of an AI-based approach that is in development in the Digital Ludeme Project (DLP) is a combination of evolutionary reconstruction of games with an evaluation of quality based on selfplay. As discussed in Section 2, there is often some information about rulesets or equipment used in games thanks to archaeological evidence, but this data tends to be incomplete. Given partial game descriptions based on such evidence, evolutionary approaches (Browne, 2009) can be used to generate a set of full games as potential reconstructions. A variety of quantitative indicators of game quality, such as the drawishness of a game, or the average game length, or any bias in favour of one player over the other, may then be automatically estimated from self-play by a game-playing agent. The ability to easily model many games in Ludii, run them efficiently for self-play purposes, and the evolvability of its ludeme-based game descriptions, facilitate such an approach.

Based on the intuition that high-quality rulesets may be more likely to have been truly used throughout history than rulesets that lead to extreme, obvious flaws in game quality, such an evaluation can be used to generate a restricted distribution of plausible games from the available evidence. It should be noted that this approach should be applied carefully; it cannot be ruled out with certainty that rulesets considered to be "obviously poor" by today's standards were not still used by ancient civilisations. AI-based evaluations of game quality are envisioned to form new pieces of evidence, which are often inconclusive on their own, but may be combined with other sources of evidence.

Other examples of computational techniques under consideration in the DLP include the use of computational phylogenetics to generate hypotheses for how games may be related to each other based on their ludemes. This may provide clues about how games spread temporally, geographically and culturally, and can be combined with other types of data (such as known trade routes, wars, etc.).

\section{CONCLUSION}

This report briefly summarised the primary points discussed in a Dagstuhl research meeting in which the new research field of Digital Archæoludology (DAL) was defined. This field involves the use of Artificial Intelligence and related computational techniques, combined with - often sparse - archaeological evidence and other traditional sources, to generate reconstructions of, and otherwise improve insights into, how games may have been played and transferred throughout history. Research in this field has been started in the Digital Ludeme Project. In addition to contributions to the new field of DAL, the project is also expected to contribute to research fields such as General Game Playing and other areas of Artificial Intelligence - for instance through the development of the Ludii general game system.

\section{ACKNOWLEDGEMENTS}

This research is part of the European Research Council-funded Digital Ludeme Project (ERC Consolidator Grant \#771292) run by Cameron Browne at Maastricht University's Department of Data Science and Knowledge Engineering. 


\section{REFERENCES}

Browne, C. (2018). Modern techniques for ancient games. In 2018 IEEE Conference on Computational Intelligence and Games (CIG 2018) (pp. 490-497). IEEE.

Browne, C. (2019). AI for ancient games. Künstliche Intelligenz. In press.

Browne, C.B. (2009). Automatic generation and evaluation of recombination games. PhD thesis, Faculty of Information Technology, Queensland University of Technology, Queensland, Australia.

Browne, C., Soemers, D.J.N.J., Piette, É., Stephenson, M., Conrad, M., Crist, W., Depaulis, T., Duggan, E., Horn, F., Kelk, S., Lucas, S.M., Neto, J.P., Parlett, D., Saffidine, A., Schädler, U., Silva, J.N., de Voogt, A. \& Winands, M.H.M. (2019). Foundations of Digital Archæoludology. https://arxiv.org/ abs/1905.13516.

Crist, W. (2019). Playing against complexity: Board games and social strategy in Bronze Age Cyprus. Journal of Anthropological Archaeology, 55.

de Voogt, A. (1997). Mancala Board Games. London: The British Museum Press.

de Voogt, A. (2003). Hawalis in Oman: A first account of expertise and dispersal of four-row Mancala in the Middle East. In Board Game Studies 6 (pp. 95-98).

Herskovits, M. (1932). Wari in the New World. The Journal of the Royal Anthropological Institute of Great Britain and Ireland, 62, 23-37. doi:10.2307/2843876.

Kowalski, J., Maksymilian, M., Sutowicz, J. \& Szykuła, M. (2019). Regular boardgames. In The Thirty-Third AAAI Conference on Artificial Intelligence (AAAI 2019) (pp. 1699-1706).

Love, N., Hinrichs, T., Haley, D., Schkufza, E. \& Genesereth, M. (2008). General game playing: Game description language specification. Stanford University.

Malaby, T. (2003). Gambling Life: Dealing in Contingency in a Greek City. Urbana, IL: University of Illinois Press.

Parlett, D. (2016). What's a Ludeme? In Game Puzzle Design (Vol. 2, pp. 83-86).

Piccione, P. (1990). The historical development of the game of Senet and its significance for Egyptian religion. PhD thesis, Department of Near Eastern Languages and Civilizations, University of Chicago, Chicago, IL, USA.

Piette, É., Stephenson, M., Soemers, D.J.N.J. \& Browne, C. (2019a). An empirical evaluation of two general game systems: Ludii and RBG. In 2019 IEEE Conference on Games (COG 2019). In press.

Piette, É., Soemers, D.J.N.J., Stephenson, M., Sironi, C.F., Winands, M.H.M. \& Browne, C. (2019b). Ludii - The Ludemic General Game System. https://arxiv.org/abs/1905.05013.

Stephenson, M., Piette, É., Soemers, D.J.N.J. \& Browne, C. (2019). An overview of the Ludii general game system. In 2019 IEEE Conference on Games (COG 2019). In press.

Tait, W.J. (1982). Game Boxes and Accessories from the Tomb of Tut'ankhamun. Tut'ankhamun's Tomb Series (Vol. VII). Oxford: Griffith Institute.

Townshend, P. (1982). Games in culture: A contextual analysis of the Swahili board game and its relevance to variation in African manual. PhD thesis, Department of Social Anthropology, Cambridge University, Cambridge. 